(1)

CrossMark

\title{
Pulmonary arterial hypertension: screening challenges in systemic sclerosis and future directions
}

\author{
Nermin Diab ${ }^{1}$ and Paul M. Hassoun ${ }^{2}$
}

Affiliations: ${ }^{1}$ Bloomberg School of Public Health, The Johns Hopkins University, Baltimore, MD, USA. ${ }^{2}$ Division of Pulmonary and Critical Care Medicine, Dept of Medicine, The Johns Hopkins University, Baltimore, MD, USA.

Correspondence: Paul M. Hassoun, Division of Pulmonary and Critical Care Medicine, 1830 East Monument Street, Baltimore, MD 21287, USA. E-mail: phassounQjhmi.edu

@ERSpublications

Screening for PAH is significant in scleroderma as it may lead to early diagnosis and initiation of therapy http://ow.ly/TR6230aqRgq

Cite this article as: Diab N, Hassoun PM. Pulmonary arterial hypertension: screening challenges in systemic sclerosis and future directions. Eur Respir J 2017; 49: 1700522 [https://doi.org/10.1183/13993003.005222017].

Despite the advent of multiple medical therapies for pulmonary arterial hypertension (PAH), there is currently no cure available for this disease which continues to impose a very heavy burden of morbidity and mortality on afflicted patients. Survival on medical therapy is at best 7 years in patients with idiopathic PAH [1] and significantly less in patients with associated PAH (APAH), such as those with connective tissue disease (CTD) and particularly with scleroderma-associated PAH (SSc-PAH), where median survival is about 4 years.

In this context and considering the unduly high delay (in the order of years) between initial symptoms and diagnosis (typically obtained by right-heart catheterisation (RHC)), it becomes important to find strategies to detect the disease as early as possible. For obvious reasons, early detection is only feasible in populations at risk for the disease, such as those with APAH (e.g., PAH complicating CTD or HIV) and other diseases from groups 2 (e.g., heart failure) and 3 (owing to lung disease) of the pulmonary hypertension $(\mathrm{PH})$ classification. Among these "diseases at risk for $\mathrm{PAH}$ ", scleroderma (SSc) is quite unique in two important aspects: 1 . the prevalence of PAH in a general cohort of SSc patients is quite high (8-12\%) compared to other similar "at risk" diseases (e.g., the prevalence of PAH in HIV is less than $1 \%)$ and, 2. when afflicted with $\mathrm{PAH}$, the survival of these patients is significantly curtailed compared to SSc patients who remain free from pulmonary vascular disease. Deaths due to PAH and pulmonary fibrosis, often complicated by $\mathrm{PH}$, together account for up to $60 \%$ of all SSc-related deaths [2].

The importance of PAH detection in SSc patients is thus obvious and early diagnosis and initiation of appropriate $\mathrm{PAH}$-specific therapy might potentially limit disease progression and improve survival rates. The PHAROS [3] and the ItinerAIR [4] SSc registries have both demonstrated that screening of SSc patients allows for early detection and potentially results in a significantly higher 3-year survival rate [4]. Whilst the best screening approach for SSc-PAH remains debatable, there is general expert consensus that screening should be considered in SSc patients on their initial visit with re-evaluation during interval visits thereafter. The current 2015 European Society of Cardiology/European Respiratory Society (ESC/ERS) guidelines [5], as compared to the 2009 guidelines, recommend that all patients with SSc-spectrum disorders be screened

Received: March 132017 | Accepted: March 142017

Conflict of interest: None declared.

Copyright OERS 2017 
for PAH at initial evaluation with pulmonary function tests including single-breath diffusing capacity of the lung for carbon monoxide (DLCO), transthoracic echocardiography and N-terminal pro-brain natriuretic peptide (NT-proBNP) measurement. Guidelines for RHC now require either a tricuspid regurgitant velocity (TRV) of $>3.4 \mathrm{~m} \cdot \mathrm{s}^{-1}$ or of $2.8-3.4 \mathrm{~m} \cdot \mathrm{s}^{-1}$ in the presence of symptoms.

The DETECT study by Coghlan [6] was the first to address the issue of missed PAH diagnoses in 466 high-risk SSc patients (DLCO $<60 \%$; duration of disease $>3$-years). A DETECT two-step-algorithm outperformed the earlier 2009 ESC/ERS guidelines as a screening tool for RHC and demonstrated the limited utility of two components of the 2009 guidelines: symptoms (dyspnoea) which did not discriminate between PAH and PH and TRV alone, which would have missed $20 \%$ of PAH patients when using a PAH suspicion threshold of $\geqslant 2.5 \mathrm{~m} \cdot \mathrm{s}^{-1}, 36 \%$ of PAH patients with a threshold of $>2.8 \mathrm{~m} \cdot \mathrm{s}^{-1}$ and $63 \%$ of PAH patients with a threshold of $>3.4 \mathrm{~m} \cdot \mathrm{s}^{-1}$. When applied to the DETECT cohort, the $2009 \mathrm{ESC} /$ ERS guidelines missed $29 \%$ of PAH diagnoses compared to $4 \%$ using the DETECT tool, a considerable difference in the false-negative rate [6].

In this issue of the European Respiratory Journal, VANDECASTEELE et al. [7] prospectively compare the 2009 ESC/ERS guidelines to the DETECT algorithm in an unselected consecutive cohort of SSc patients irrespective of their risk factors or extent of skin involvement, a real-life clinical situation of a diverse population followed at an SSc referral centre at Ghent University Hospital in Belgium. In their cohort of 195 patients, out of 63 patients referred for RHC, referral was 46 (73\%) using the DETECT algorithm alone, four (6\%) using the 2009 ESC/ERS guidelines alone, and 34 (17\%) and 40 (21\%) using the 2015 echo-screening and 2015 combined-screening guidelines, respectively, in a post-hoc analysis. Only three patients were diagnosed with $\mathrm{PAH}$, with RHC recommended by all of the examined screening algorithms. The results demonstrated a positive predictive value of $6 \%$ using the DETECT algorithm compared to $18 \%$ using the 2009 guidelines (and 11\% and 9\% using the 2015 echo-screening and combined-screening guidelines, respectively). The authors demonstrate improved cost-effectiveness of the 2009 guidelines compared to the DETECT tool, concluding that echocardiography remains the preferred first step for PAH screening in SSc patients.

To date, the DETECT algorithm has not been validated in patients with a DLCO value of $>60 \%$ of predicted and such patients constitute more than $75 \%$ of the Vandecasteele cohort (median $=76 \%$; interquartile range: $63.25-87$ ). It is therefore not surprising that the positive predictive values of patients referred for RHC by the 2009 guidelines and the 2015 echo-screening and combined-screening guidelines are greater than that of the DETECT algorithm in this unselected group of SSc patients. This study demonstrates the superior screening performance, from a cost-analysis point of view, of the 2009/2015 guidelines (compared to DETECT) regardless of SSc patients' risk status and also their reliability for specific detection of PAH. Some obvious limitations of the study include the lack of data on sensitivity, specificity and the negative predictive value of the screening algorithms (since RHC was not applied to the whole cohort), the paucity of patients diagnosed with $\mathrm{PAH}$ (a total of three), and the post-hoc analysis of the 2015 ESC/ERS guidelines (as these were only published after initiation of the study [5]).

This study further emphasises the value of echocardiography in screening patients with SSc. Echocardiography is readily available although it has its own important limitations, including high dependency on the operator/interpreter, over-estimation or under-estimation of systolic pulmonary arterial pressure (PAP) [8], and false-negative and false-positive results. In this respect, GLADUE [9] has demonstrated that the use of echocardiography alone fails to diagnose PAH in up $31 \%$ of subjects in a large cohort of SSc patients, half of whom were eventually captured by combining echocardiographic results with pulmonary function tests. Furthermore, tricuspid regurgitation may be absent in a number of patients with $\mathrm{PH}$, as suggested by the DETECT study in which it was not appreciable for 7\% of patients with SSc-PAH [6]. Finally, echocardiography in SSc-PAH evaluation cannot differentiate between different types of $\mathrm{PH}$, an important step in treatment strategies.

One noteworthy finding by Vandecasteele et al. relates to the fact that 13 out of 14 cases (93\%) of borderline elevated PAP were detected by RHC as recommended by the DETECT algorithm versus only four out of 14 cases (29\%) being detected by RHC following the 2009 guidelines. In light of the PHAROS registry finding that $55 \%$ of patients with borderline elevated PAP on initial RHC go on to develop PH (but not necessarily $\mathrm{PAH}$ ) over a follow-up period of 2 years [10], this may be an advantage in favour of the DETECT algorithm.

Finally, is early detection for more aggressive therapy in SSc-PAH justifiable? This question may have seemed overly futile just a few years ago as most large clinical trials for PAH therapies demonstrated at best a modest clinical response in SSc-PAH compared to idiopathic pulmonary arterial hypertension (IPAH). However, this state of affairs may have changed with more recent trials such as the phase III PATENT-1 study (PATENT: Pulmonary Arterial hyperTENsion sGC-stimulator Trial). In this trial riociguat, a guanylate 
cyclase stimulator given to patients with CTD associated PAH, was found to improve primary end-point (6-min walk distance), World Health Organization (WHO) functional class, and haemodynamics compared to a placebo treatment [11]. In addition, the 2-year survival rate was similar (93\%) for patients with CTD associated PAH compared to IPAH [12]. Of note is the fact that about $63 \%$ of patients with CTD associated $\mathrm{PAH}$ were on background therapy with an endothelin receptor antagonist or prostacyclin [12]. More recently, the AMBITION trial (a uniquely designed protocol which used upfront combination therapy with two U.S. Food \& Drug Administration (FDA) approved drugs, ambrisentan and tadalafil, for PAH [13]) demonstrated that time to clinical worsening in patients with PAH was improved. This was mainly driven by differences in hospitalisation rates (due to worsening PAH) between either of the monotherapies and the combination therapy. In a post-hoc analysis, initial combination therapy reduced the risk of clinical failure versus pooled monotherapy in each subgroup of CTD associated PAH, including SSc-PAH [14].

In conclusion, efforts towards early detection of PH in SSc are worthwhile and further studies, such as the one elegantly performed by VANDECASTEELE et al. [7], are needed to validate the various algorithms available in the specific context of an unselected SSc population. While RHC is the gold-standard for the diagnosis of PH in SSc, echocardiography remains a well-anchored screening tool for pulmonary vascular disease under these circumstances [15].

\section{References}

1 Benza RL, Miller DP, Barst RJ, et al. An evaluation of long-term survival from time of diagnosis in pulmonary arterial hypertension from the REVEAL registry. Chest 2012; 142: 448-456.

2 Steen VD, Medsger TA. Changes in causes of death in systemic sclerosis, 1972-2002. Ann Rheum Dis 2007; 66: 940-944.

3 Hinchcliff M, Fischer A, Schiopu E, et al. Pulmonary Hypertension Assessment and Recognition of Outcomes in Scleroderma (PHAROS): baseline characteristics and description of study population. J Rheumatol 2011; 38: 2172-2179.

4 Humbert M, Yaici A, de Groote P, et al. Screening for pulmonary arterial hypertension in patients with systemic sclerosis: clinical characteristics at diagnosis and long-term survival. Arthritis Rheum 2011; 63: 3522-3530.

5 Galiè N, Humbert M, Vachiery JL, et al. 2015 ESC/ERS Guidelines for the diagnosis and treatment of pulmonary hypertension. Eur Respir J 2015; 46: 903-975.

6 Coghlan JG, Denton CP, Grünig E, et al. Evidence-based detection of pulmonary arterial hypertension in systemic sclerosis: the DETECT study. Ann Rheum Dis 2014; 73: 1340-1349.

7 Vandecasteele E, Drieghe B, Melsens K, et al. Screening for pulmonary arterial hypertension in an unselected prospective systemic sclerosis cohort. Eur Respir J 2017; 49: 1602275.

8 Fisher MR, Criner GJ, Fishman AP, et al. Estimating pulmonary artery pressures by echocardiography in patients with emphysema. Eur Respir J 2007; 30: 914-921.

9 Gladue H, Steen V, Allanore Y, et al. Combination of echocardiographic and pulmonary function test measures improves sensitivity for diagnosis of systemic sclerosis-associated pulmonary arterial hypertension: analysis of 2 cohorts. J Rheumatol 2013; 40: 1706-1711.

10 Bae S, Saggar R, Bolster MB, et al. Baseline characteristics and follow-up in patients with normal haemodynamics versus borderline mean pulmonary arterial pressure in systemic sclerosis: results from the PHAROS registry. Ann Rheum Dis 2012; 71: 1335-1342.

11 Ghofrani HA, Galiè N, Grimminger F, et al. Riociguat for the treatment of pulmonary arterial hypertension. N Engl J Med 2013; 369: 330-340.

12 Humbert M, Coghlan JG, Ghofrani HA, et al. Riociguat for the treatment of pulmonary arterial hypertension associated with connective tissue disease: results from PATENT-1 and PATENT-2. Ann Rheum Dis 2017; 76: 422-426.

13 Galiè N, Barberà JA, Frost AE, et al. Initial use of Ambrisentan plus Tadalafil in pulmonary arterial hypertension. N Engl J Med 2015; 373: 834-844.

14 Coghlan JG, Galiè N, Barberà JA, et al. Initial combination therapy with Ambrisentan and Tadalafil in connective tissue disease-associated pulmonary arterial hypertension (CTD-PAH): subgroup analysis from the AMBITION trial. Ann Rheum Dis 2016; in press [https://doi.org/10.1136/annrheumdis-2016-210236].

15 Grünig E, Peacock AJ. Imaging the heart in pulmonary hypertension: an update. Eur Respir Rev 2015; 24: 653-664. 